\title{
Langerhans Cells Express IL-17A in the Epidermis of Chromoblastomycosis Lesions
}

\author{
Aline Alves de Lima Silva ${ }^{a}$ b $\quad$ Paulo Ricardo Criado ${ }^{b}$ Ricardo Spina Nunes ${ }^{b}$ \\ Luciane Kanashiro-Galo ${ }^{a}$ Maria Irma Seixas Duarte ${ }^{a}$ Mirian N. Sotto ${ }^{a}$ b \\ Carla Pagliaria, b \\ a Laboratório da Disciplina de Patologia de Moléstias Transmissíveis/Departamento de \\ Patologia-Faculdade de Medicina da Universidade de São Paulo, and bepartamento de \\ Dermatologia - Faculdade de Medicina da Universidade de São Paulo, São Paulo, Brazil
}

\begin{abstract}
What Is It about?
Chromoblastomycosis (CBM) is an important disease in tropical regions and is responsible for much biopsychosocial damage in patients. Our study presented for the first time the coexpression of IL-17 and Langerin in dendritic cells in the epidermis and dermis of lesions, which is not seen in healthy patients. Dendritic cells in CBM play an important role both as antigen-presenting cells and in the elimination of fungi. The immune response is also marked by the imbalance in Th17 response. We hope that our data can contribute to a better understanding of the disease, leading to more effective therapies for CBM.
\end{abstract}

\section{Keywords}

Chromoblastomycosis · Skin lesions · Langerhans cells · Langerin · Th17

Abstract

Chromoblastomycosis (CBM) is a chronic fungal infection that affects skin and subcutaneous tissue, and little is known about the immunological aspects of such lesions. We have previously described the high expression of IL-17 in this group. Understanding the innate immune response of patients with CBM would improve the knowledge of its immunopathogenesis and contribute to the most appropriate therapies. Nineteen biopsies of verrucous form were obtained from patients with clinical and histopathological diagnosis of CBM, without treatment. This was done with a double immunostaining with conventional immunohistochemistry and immunofluorescence technique as well as confocal microscopy to detect Langerin and IL-17 expression. All of the specimens that were analyzed showed expression of Langerin in the epidermis - the same as the control group. However, only the CBM group presented cells expressing CD207 in the dermis. Interestingly, the coexpression of IL-17 and Langerin was visualized along the epidermis and dermis in $100 \%$ of the lesion group. We demonstrated for the 
first time in situ coexpression of IL-17 and Langerin (CD207) in epidermal cells of patients with CBM and speculated on their role as IL-17-producing cells or whether they could be a new subpopulation of dendritic cells distinct from Langerhans cells.

(C) 2017 The Author(s)

Published by S. Karger AG, Basel

\section{Introduction}

Chromoblastomycosis (CBM) is a chronic fungal infection present worldwide and with remarkable incidence in tropical and subtropical countries [1]. The disease is caused by dimorphic dematiaceous fungi. The lesions usually affect the skin and subcutaneous tissue of the lower limbs of rural workers $[1,2]$.

Langerhans cells (LCs) constitute a subclass of dendritic cells of the skin and mucosa. They are characterized by the presence of Birbeck granules and Langerin (CD207), a specifically expressed C-type lectin receptor [3]. The role of Langerin in fungal infections is not fully elucidated. LCs can phagocytose Fonsecaea pedrosoi sclerotic cells and inhibit fungi hyphae formation [4].

LCs, as well as macrophages and FXIIIa+ dermal dendrocytes, play a role in fungi antigen presentation and immunomodulation in CBM human lesions [5]. Thus, at least in the pathogenesis of CBM, LCs seem to be involved both in innate and dynamic adaptive responses.

When Th1 and Th2 patterns of cytokines are ineffective, another pattern of immune response seems to get established as an immune booster: the Th17 cells. These are mostly CD4+ cells characterized by the expression of proinflammatory cytokines, especially IL-17 and IL-23 [6, 7]. Th17 lymphocytes have been detected as protagonists in chronic inflammatory microenvironments and there are currently many studies about their development and functions [6].

Recently, a significant presence of IL-17+ cells in human lesions of CBM has been shown. These cells could represent an attempt to help the primary immune profile against the fungi. However, it is possible that the marked imbalance between the effectors and regulatory profiles contribute to the ineffective response against verrucous CBM [8].

Interestingly, dendritic IL-17+ cells have been observed in the epidermis and dermal infiltrate in human CBM verrucous lesions [8]. In order to verify whether these IL-17+-expressing dendritic cells are in fact LCs, we studied CD207 and IL-17 coexpression by double immunolabeling CBM lesions.

\section{Materials and Methods}

\section{Biopsies}

Nineteen biopsies of CBM lesions were retrieved from the files of the Dermatopathology Laboratory (Division of Clinical Dermatology, Hospital das Clinicas, Faculty of Medicine, University of São Paulo). The biopsies were taken from patients with CBM verrucous lesions, sclerotic bodies at histopathological exam, and F. pedrosoi in culture (84.3\% males, mean age 62.5 years old). Ten normal skin fragments constituted the control group.

The Ethics Committee of Clinical Hospital, Faculty of Medicine of University of São Paulo approved the study (protocol No. 0317/11).

\section{Histopathological Analysis}

All the hematoxylin-eosin-stained biopsies were reviewed in order to confirm the CBM diagnosis. 


\section{Immunohistochemistry}

4- $\mu \mathrm{m}$ paraffin-embedded fragments of each biopsy were deparaffinized and hydrated in ethanol, and subjected to antigen retrieval in TRIS/EDTA buffer pH 9.0 for $20 \mathrm{~min}$ at $95^{\circ} \mathrm{C}$. The primary antibodies anti-IL-17 (clone IL-17A) and CD207 (clone 12D6) were diluted in $1 \%$ bovine albumin solution and incubated overnight at $4{ }^{\circ} \mathrm{C}$. This was followed by the second antibody incubation and streptavidin-peroxidase complex. The reaction was developed with 3,3-diaminobenzidine tetrahydrochloride (DAB) chromogen and the slides were counterstained lightly with hematoxylin. All reactions were performed with positive (normal skin and palatine tonsil fragments) and negative controls. The negative controls of the reaction were obtained by replacing the primary antibodies by isotypic nonreagent antibodies.

Double Immunostaining with Conventional Immunohistochemistry and

Immunofluorescence Technique and Confocal Microscopy

The initial procedures were similar as described above. The detection of Langerin + cells was performed with DAB as described above, followed by incubation with anti-IL-17 antibody and a streptavidin-biotin phosphatase developing system and permanent red chromogen.

Briefly, the immunofluorescence technique protocol for confocal microscopy was performed as follows: the antibodies anti-IL-17 (clone IL-17A) and anti-Langerin (clone 12D6) were diluted in $1 \%$ bovine albumin solution and incubated for $48 \mathrm{~h}$ at $4^{\circ} \mathrm{C}$. After this, isotype-specific secondary conjugated antibodies (Alexa Fluor 488 and Alexa Fluor ${ }^{\circledR} 546$, Invitrogen, Carlsbad, CA, USA) were applied and nuclear staining with 4',6-diamidino2-phenylindole, dihydrochloride (DAPI; Sigma-Aldrich, Steinhein, Germany) was done for 60 min. The slides were mounted in mounting medium (Hydromount; National Diagnostics, Atlanta, GA, USA) and on glass coverslips, and maintained protected from light at $4^{\circ} \mathrm{C}$ until analysis. Fluorescent images were acquired using UV/laser excitation on a LSM/Meta 510 Zeiss microscope and LSM Image Examiner software (Carl Zeiss, Standort Gottingen, Germany) at the Confocal "Rede Premium" Multiuser Facility of the Heart Institute of São Paulo University.

Quantitative Analysis

The Langerin+ cells visualized through single-staining procedure were quantified by counting the positive stained epidermal area fraction using a $\times 10$ ocular lens with a square grid and a $\times 40$ objective [9]. The IL-17+ cells were quantified by counting the number of immunolabeled cells in 9 randomized high-power fields for each specimen with a $\times 10$ ocular lens with a square grid area of $0.0625 \mathrm{~mm}^{2}$.

\section{Statistical Analysis}

The expression of CD207 both in CBM and control epidermis was compared using Graph Pad Prism version 5.00 for Windows (Graph Pad Software, San Diego, CA, USA) to perform the Mann-Whitney test with the level for significance set at 95\% $(p \leq 0.05)$.

\section{Results}

\section{Histopathological Analyses}

All the CBM lesions displayed epidermal hyperplasia with hyperkeratosis, parakeratosis, and acanthosis with irregular epidermal elongations. The dermis exhibited granulomatous infiltrate with neutrophils exudate foci, mainly in the granuloma center. There was variable number of dematiaceous yeast cells phagocytosed by giant cells or in granuloma exudative foci. 


\section{Expression of Langerin (CD207) and IL-17}

All of the CBM specimens studied displayed CD207+ dendritic cells in the epidermis and among the dermal inflammatory infiltrate. The positive cells were distributed throughout the epidermis and also the dermis (Fig. 1a, c). The frequency of CD207+ cells varied from mild to moderate intensity. The normal skin biopsies displayed CD207+ dendritic cells among keratinocytes in the epidermis. In the dermis, these cells were very scant or even absent (Fig. 1b, d).

IL-17+ cells were observed in all CBM specimens. These cells were distributed in the dermis among the inflammatory infiltrate. The epidermis displayed few IL-17+ dendritic and spindle cells (Fig. 1e, g). Biopsies from the control group displayed only a few IL-17-expressing cells in the dermis (Fig. 1f, h).

\section{Coexpression of CD207 and IL-17 through Double-Labeling Immunohistochemistry and}

Immunofluorescence Technique

The exam of biopsies subjected to both techniques displayed coexpression of CD207 and IL-17 in some epidermal and dermal dendritic cells in CBM lesions (Fig. 2a-g). None of the normal skin group displayed double-labeling cells in the epidermis or dermis.

\section{Discussion}

The skin is the first barrier to pathogens, both physically and immunologically, and in CBM it is also the structure by which the fungus presents tropism [1].

The immunological cascade begins with the capture and presentation of antigens, a masterful activity of LCs [10]. Langerin (CD207) is a marker of mature LCs [3]. Nineteen skin biopsies of verrucous type were analyzed and diagnosed as CBM by positive culture of $F$. pedrosoi and clinical analysis.

In CBM the immune response is mediated mainly by Th2 cytokines [11]. Previously we demonstrated that in CBM lesions a predominance of Th17 cells over Treg cells occurs [8]. The imbalance between the regulator and effector profiles could be a major contributor to the inefficiency of the immune response established in this mycosis.

The epidermal CD207 expression in CBM lesions was lower when compared to normal skin ( $p=0.0001)$. Otherwise, $68.42 \%$ of CBM specimens displayed CD207-expressing cells among the dermal inflammatory infiltrate, and these cells were almost absent in the dermis of the normal skin group.

The presence of CD207+ cells in the CBM dermis led us to speculate that it may be due to migration of LCs in the way to present fungi antigens at the lymph nodes. There is speculation whether CD207-expressing cells are in fact LCs, or another population of dendritic cells, mainly in the dermal compartment [12]. Langerin-expressing cells were frequently observed in the dermis of patients affected by paracoccidioidomycosis [13]. CD207-positive dermal cells may exert a role in repopulation, which does not seem to be played by the LCs; furthermore, dermal Langerin+ cells usually grow up in microenvironments where LCs are absent [14].

Fig. 1. Immunoexpression of Langerin (CD207) and IL-17 in the chromoblastomycosis (CBM) skin lesion and control groups. a, c Expression of CD207 in epidermal cells in CBM lesions. b, d expression of CD207 in specimens of normal skin. e, $\mathbf{g}$ Note numerous immunoreactive IL-17A+ cells in the epidermis. They are of similar morphology as Langerin+ cells (compare $\mathbf{a}$ and $\mathbf{b}$ ). The dermis also contains IL-17A+ cells, presumably $\mathrm{T}$ cells. $\mathbf{f}$, $\mathbf{h}$ Expression of IL-17A+ cells in normal skin; note the presence of these cells in the dermis, which probably are sentinels of skin immune system (SIS) (streptavidin-biotin peroxidase in $\times 400$ ).

(For figure see next page.) 


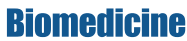 紏们}

2017 The Author(s). Published by S. Karger AG, Basel www.karger.com/bmh

Alves de Lima Silva et al.: Langerhans Cells Express IL-17A in the Epidermis of

CBM
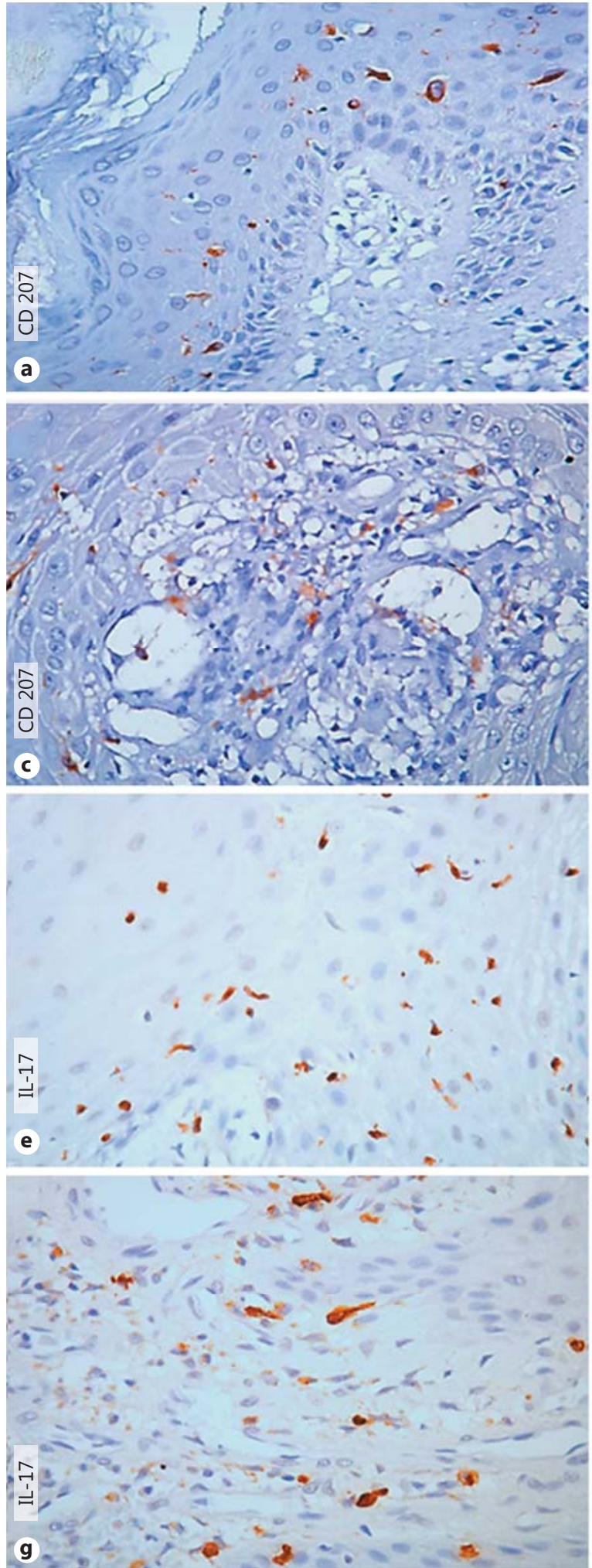

\section{Normal skin}
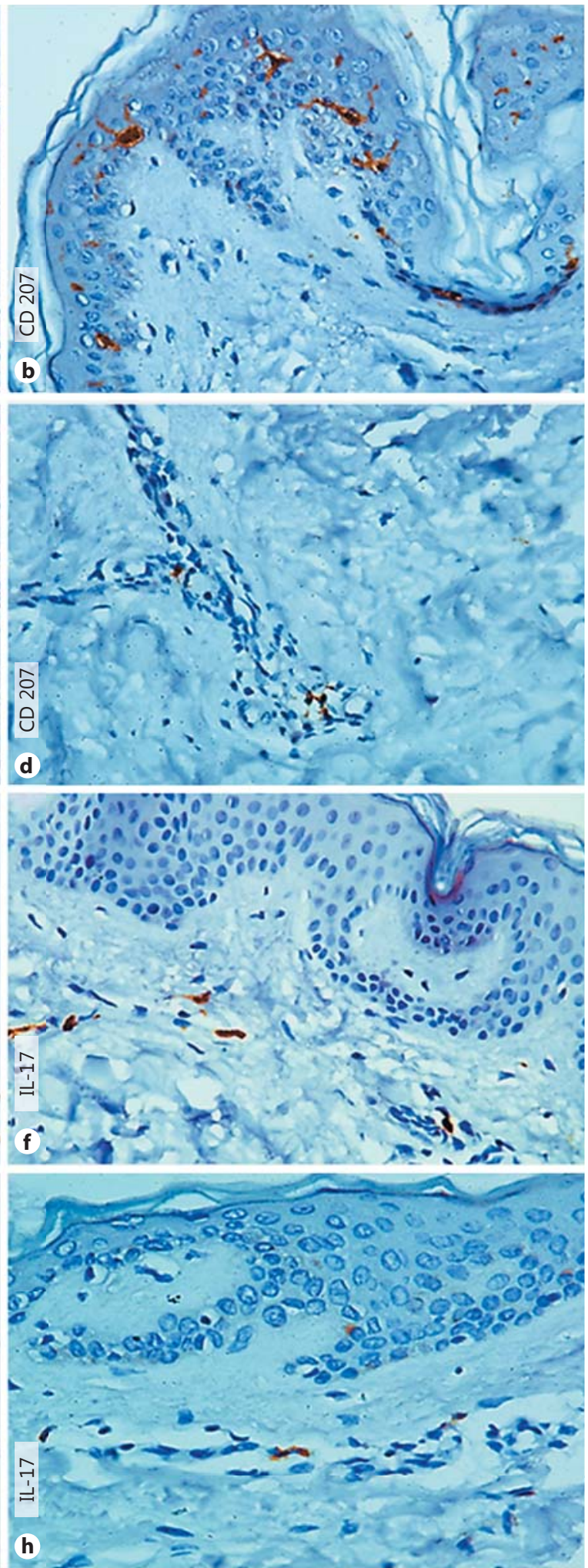

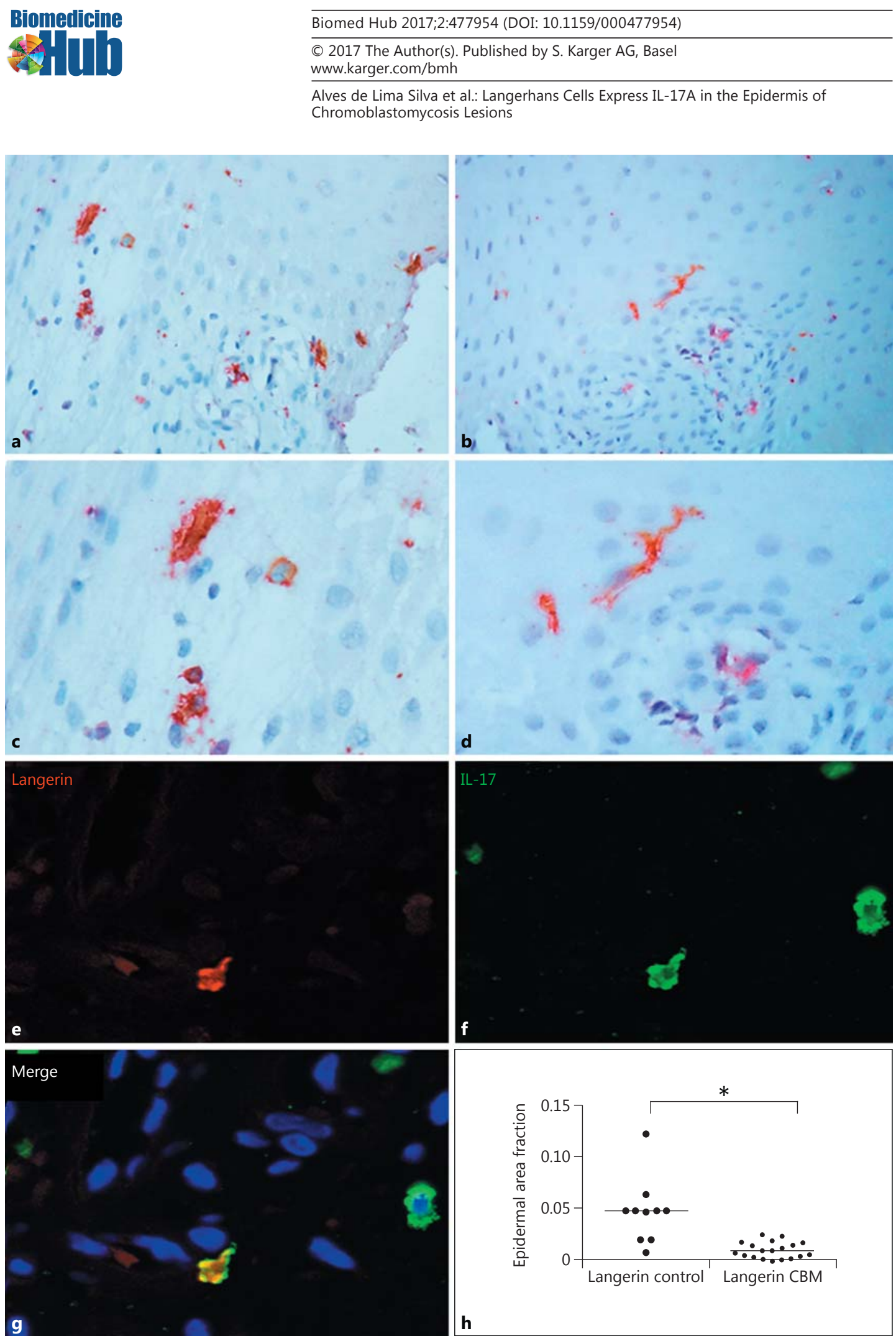

Fig. 2. Double immunostaining (conventional and confocal) demonstrating coexpression of Langerin/IL-17. Peroxidase-labeled streptavidin-biotin detection of Langerin was evidenced in brown staining; alkaline phosphatase-labeled detection of IL-17 was evidenced in red. Note the presence of immunostaining cells in the dermis ( $\mathbf{a}$ and $\mathbf{b}$, magnifications $\times 400$, details on $\mathbf{c}$ and $\mathbf{d}$ ). e Detection of Langerin was evidenced in orange staining (546). $\mathbf{f}$ Detection of IL-17 was evidenced in green (488). $\mathbf{g}$ Demonstration of merge with nuclear staining in blue (DAPI) (magnifications $\times 200$ ). $\mathbf{h}$ Comparative analysis of Langerin among the chromoblastomycosis (CBM) and normal skin groups: distribution of number of cells/epidermal area fraction expressing Langerin. Mann-Whitney test, ${ }^{*} p<0.0001$. 
In addition to our previous report that demonstrated positive IL-17 dermal cells in the inflammatory infiltrates, a more detailed analysis of IL-17 expression in CBM lesions allowed us herein to identify the positive immunoreactivity of dendritic cells in the epidermis. The double-staining immunohistochemistry technique and immunofluorescence with confocal microscopy have shown that CD207-expressing cells in the epidermis coexpress IL-17 in CBM lesions.

IL-17 expression by CD207-positive dendritic cells was previously described by Coury et al. [15] in skin and bone lesions of LC histiocytosis. Blood monocytes of LC histiocytosis patients also produce IL-17 [16].

Similar to the results of Bursch et al. [12], we previously demonstrated that CBM lesions display low expression of TGF- $\beta$ [8], which could reinforce the hypothesis that CD207-positive dermal cells are a new subpopulation of distinct dendritic cells and that, at least in our study, they seem to cooperate with the cascade of the Th17 immune response $[12,14]$.

In order to avoid misunderstandings, the analysis of our data took place at 3 different times: analysis of both IL-17 and CD207 markers through simple immunohistochemistry followed by the double immunohistochemistry technique and validation of the results with immunofluorescence technique and confocal microscopy.

The authors feel that the data presented herein may contribute to the understanding of the role of LCs as expressing IL-17 in the immunopathogenesis of CBM. In addition, the cells expressing CD207 could constitute new subclass of this lineage of dendritic cells.

\section{Acknowledgement}

We thank Prof. Nikolaus Romani from Innsbruck Medical University for his critical reading of this manuscript.

\section{Ethics Statement}

The use of the material that constituted the casuistry was approved by the Ethics Committee of Clinical Hospital, Faculty of Medicine of University of São Paulo (No. 0317/11).

\section{Disclosure Statement}

The authors have no conflicts of interest.

\section{Funding}

This work was supported by the FAPESP - Fundação de Apoio à Pesquisa do Estado de São Paulo; São Paulo, Brasil (grant No. 2013/07994-1). 
(C) 2017 The Author(s). Published by S. Karger AG, Basel www.karger.com/bmh

Alves de Lima Silva et al.: Langerhans Cells Express IL-17A in the Epidermis of Chromoblastomycosis Lesions

\section{References}

1 Queiroz-Telles F, Esterre P, Perez-Blanco M, et al: Chromoblastomycosis: an overview of clinical manifestations, diagnosis and treatment. Med Mycol 2009;47:3-15.

$\checkmark 2$ Ibrahim-Granet $\mathrm{O}$, de Bievre C, Jendoubi M: Immunochemical characterization of antigens and growth inhibition of Fonsecaea pedrosoi by species-specific IgG. J Med Microbiol 1998;26:217-222.

-3 Valladeau J, Ravel O, Dezutter-Dambuyant C, et al: Langerin, a novel C-type lectin specific to Langerhans cells, is an endocytic receptor that induces the formation of Birbeck granules. Immunity 2000;12:71-81.

-4 da Silva JP, da Silva MB, Salgado UI, et al: Phagocytosis of Fonsecaea pedrosoi conidia, but not sclerotic cells caused by Langerhans cells, inhibits CD40 and B7-2 expression. FEMS Immunol Med Microbiol 2007;50:104111.

5 Sotto MN, De Brito T, Silva AM, et al: Antigen distribution and antigen-presenting cells in skin biopsies of human chromoblastomycosis. J Cutan Pathol 2004;31:14-18.

6 Weaver CT, Hatton RD, Mangan PR, et al: IL-17 family cytokines and the expanding diversity of effector T cell lineages. Annu Rev Immunol 2007;25:821-852.

7 Yang Y, Xu J, Niu Y, et al: T-bet and eomesodermin play critical roles in directing T cell differentiation to Th1 versus Th17. J Immunol 2008;181:8700-8710.

8 Silva AAL, Criado PR, Nunes RS, et al: In situ immune response in human chromoblastomycosis: a possible role for regulatory and Th17 T cells. PLoS Negl Trop Dis 2014;8:e3162.

-9 Bieber T, Ring J, Braun-Falco 0: Comparison of different methods for enumeration of Langerhans cells in vertical cryosections of human skin. Br J Dermatol 1988;118:385-392.

10 Banchereau J, Steinman RM: Dendritic cells and the control of immunity. Nature 1998;392:245-252.

11 D'Avila SC, Pagliari C, Duarte MI: The cell-mediated immune reactions in cutaneous lesions of chromoblastomycosis and their correlation with different clinical forms of the diseases. Mycopathologia 2003;156:51-60.

12 Bursch LS, Wang L, Igvarto B, et al: Identification of a novel population of Langerin+ dendritic cells. J Exp Med 2007;204:3147-3156.

13 Pagliari C, Fernandes ER, da Silva WLF, et al: Revisiting Langerhans cells in paracoccidioidomycosis: expression of CD207/Langerin in human cutaneous and mucosal lesions. Microb Infect 2011;13:1012-1017.

-14 Romani N, Clausen BE, Stoitzner P: Langerhans cells and more: Langerin-expressing dendritic cell subsets in the skin. Immunol Rev 2010;234:120-141.

15 Coury F, Annels N, Rivollier A, et al: Langerhans cell histiocytosis reveals a new IL-17 A-dependent pathway of dendritic cell fusion. Nat Med 2008;14:81-87.

$\checkmark 16$ Lourda M, Olsson-Åkefeldt S, Gavhed D, et al: Detection of IL-17A-producing peripheral blood monocytes in Langerhans cell histiocytosis patients. Clin Immunol 2014;153:112-122. 